\title{
Forma de fuste de árvores de Eucalyptus em plantios com diferentes densidades iniciais
}

\author{
Stem form of Eucalyptus trees in plantations \\ under different initial densities
}

\author{
Rafael Ribeiro Souza', Gilciano Saraiva Nogueira², \\ Leonidas Soares Murta Júnior ${ }^{3}$, Eduardo Pelli ${ }^{4}$, Marcio Leles Romarco de Oliveira ${ }^{5}$, \\ Christovão Pereira Abrahão ${ }^{6}$ e Hélio Garcia Leite ${ }^{7}$
}

\begin{abstract}
Resumo
O objetivo deste trabalho foi avaliar o efeito do arranjo espacial de plantio na forma do fuste de árvores de eucalipto. Foram utilizados dados de um experimento implantado em dezembro de 2002, no delineamento em blocos (três blocos), sendo testados os arranjos espaciais de 3,0 x 0,5; 3,0 × 1,0;3,0 x 1,5; 3,0 × 2,0 e $3,0 \times 3,0 \mathrm{~m}$. Aos oito anos de idade foram abatidas e cubadas 180 árvores. Esses dados foram utilizados para ajustar os modelos de afilamento propostos por Garay e de Schöepfer. Testes de identidade de modelos foram aplicados nas equações com a finalidade de verificar a igualdade entre a forma dos fustes. $O$ modelo de Garay é o mais indicado para descrever o afilamento de eucalipto nos arranjos espaciais avaliados. Foi aceita a hipótese de nulidade no teste de identidade de modelos não linear, indicando a igualdade entre as equações nos arranjos de 3,0 x 0,5 e 3,0 x 1,0 m. Para uma distância de 3 metros entre fileiras, quanto maior a distância entre plantas, mais cônica é a forma do fuste.
\end{abstract}

Palavras-chave: afilamento, espaçamento, identidade de modelos.

\begin{abstract}
The objective of this study was to evaluate the effect of the spatial arrangement of planting on the stem form of eucalypt trees. Data used were from an experiment established in December 2002, in random blocks design (three blocks), with the spatial arrangements of $3,0 \times 0,5 ; 3,0 \times 1,0 ; 3,0 \times 1,5 ; 3,0 \times 2,0$ and 3,0 $x$ $3,0 \mathrm{~m}$ being tested. At eight years of age 180 trees were harvested and cubed. These data were used to adjust the taper models of Garay and Schöepfer. Model identity tests were applied on the fitted equations in order to verify the equivalence between the shapes of the boles. The Garay model is the most suitable to describe the taper of eucalypts in the evaluated spatial arrangements. The null hypothesis in the identity test of nonlinear models was accepted, indicating equality between the equations in the arrangements of $3,0 \times 0,5$ and $3,0 \times 1,0 \mathrm{~m}$. For a distance of $3,0 \mathrm{~m}$ between the rows; the greater the distance between plants the most conical shaped is the bole.
\end{abstract}

Keywords: taper, spacing, identity of models.

\section{INTRODUÇÃO}

A forma do fuste ou seu afilamento é definida pela taxa de decréscimo do diâmetro $(d)$ ao longo do fuste da árvore. Ela pode variar com a espécie ou material genético, arranjo espacial e tratos silviculturais (poda e desbaste), além da capacidade produtiva do local (CAMPOS; LEITE, 2013), e o seu estudo é feito por meio de modelos matemáticos que expressam o afilamento do fuste.

\footnotetext{
${ }^{1}$ Mestre em Ciência Florestal. UFVJM - Universidade Federal dos Vales do Jequitinhonha e Mucuri. Campus JK - Rodovia MGT 367 - Km 583, no 5000 - 39100-000 - Diamantina, MG, Brasil. E-mail: rafaelp.eflo@yahoo.com.br

2Professor Associado II. UFVJM - Universidade Federal dos Vales do Jequitinhonha e Mucuri. Campus JK - Rodovia MGT 367 - Km 583, nº 5000 - 39100-000 - Diamantina, MG, Brasil. E-mail: nogueirags@yahoo.com.br

${ }^{3}$ Doutorando em Ciências Florestais. UNB - Universidade de Brasília. Campus Darcy Ribeiro - Asa Norte - 70904970 - Brasília, DF, Brasil. E-mail: murtaengflo@gmail.com

${ }^{4}$ Mestre em Ciências Florestais - Professor Auxiliar I. UFVJM - Universidade Federal dos Vales do Jequitinhonha e Mucuri. Campus JK - Rodovia MGT 367 - Km 583, nº 5000 - 39100-000 - Diamantina, MG, Brasil. E-mail: eduardopelli@gmail.com ${ }_{5}^{5}$ Professor Adjunto IV. UFVJM - Universidade Federal dos Vales do Jequitinhonha e Mucuri. Campus JK - Rodovia MGT 367 - Km 583, nº 5000 - 39100-000 - Diamantina, MG, Brasil. E-mail: marcioromarco@gmail.com

${ }^{6}$ Professor Adjunto I. UFVJM - Universidade Federal dos Vales do Jequitinhonha e Mucuri. Campus JK - Rodovia MGT 367 - Km 583, no 5000 - 39100-000 - Diamantina, MG, Brasil. E-mail: cabrahao@gmail.com

${ }^{7}$ Professor Titular. UFV - Universidade Federal de Viçosa /Centro de Ciências Agrárias, Departamento de Engenharia Florestal. Avenida Ph Rolfs, s/n- Campus UFV - 36570-000 - Vicosa, MG, Brasil. E-mail: hgleite@gmail.com
}

Sci. For., Piracicaba, v. 44, n. 109, p. 33-40, mar. 2016

DOI: dx.doi.org/10.18671/scifor.v44n109.03 
Os modelos de afilamento são relações funcionais e constituem uma importante ferramenta estatística de uso corrente na determinação dos perfis dos fustes. Esses modelos servem para estimar três características básicas, o diâmetro $(d)$ em uma determinada altura $(h)$, para estimar a altura $(h)$ onde ocorre um diâmetro $(d)$ qualquer e o volume $(v)$ entre duas alturas quaisquer $\left(h_{1}\right.$ e $\left.h_{2}\right)$ e, permite modelar a forma do fuste da árvore ao longo de sua vida (KOZAK et al. 1969).

A variável dependente de uma função de afilamento é a razão entre o diâmetro $(d)$ a uma altura conhecida $(h)$ e o diâmetro com casca a $1,30 \mathrm{~m}($ dap). Diversos modelos de afilamento têm sido propostos e empregados para exprimir o perfil do fuste, contemplando as variações de forma. Esses modelos não descrevem todo o fuste com a mesma precisão, logo o melhor modelo dependerá dos objetivos do manejador, assim, enquanto um determinado modelo é mais eficiente em estimar $d$, ele pode não ser o melhor para estimar $h$ ou $v$. Na prática, se um modelo estima $d$ com exatidão, para qualquer $h$, não é necessário avaliá-lo em relação às estimativas de $h$ e $v$. Uma razão para um modelo ser eficiente na estimativa de $d$ e não de $h$ é que a altura não é variável dependente, para o qual se aplica o procedimento de ajuste (a minimização de erros) (CAMPOS; LEITE, 2013).

$\mathrm{O}$ arranjo inicial do plantio pode influenciar de modo significativo o crescimento das árvores e a forma de seus fustes (SCHONAU; COETZEE, 1989). Em muitos casos, para um mesmo tipo de manejo e idade, o fator de forma tende a aumentar à medida que se tem maior número de árvores por hectare no plantio (SCHONAU; COETZEE, 1989; FRIEDL, 1989; NOGUEIRA et al. 2008; RANCE et al. 2012).

Geralmente, nos plantios de eucalipto realizados no Brasil, seja com fins energéticos ou para algum setor de base da indústria, são utilizados arranjos espaciais convencionais entre 3,0 x 2,0 e 3,0 x 3,0 m, e por este motivo os estudos sobre o afilamento dos fustes se referem a estes arranjos (PIRES; CALEGARIO, 2007; SOUZA et al. 2008a; SOUZA et al. 2008b, MIGUEL et al. 2011), havendo poucos estudos relacionados à forma dos fustes em povoamentos implantados com arranjos espaciais diferentes dos convencionais.

O efeito do ambiente e das práticas silviculturais influencia diretamente na distribuição do incremento anual de madeira ao longo do tronco das árvores. Este processo fisiológico repercute diretamente na forma do fuste, que por sua vez, influencia no volume total, na qualidade e na quantidade da madeira oriunda da floresta (ANDRADE et al. 2007).

Diante do exposto, este trabalho teve como objetivo avaliar o efeito do arranjo espacial de plantio na forma do fuste de árvores de eucalipto.

\section{MATERIAL E MÉTODOS}

Foram utilizados dados de um experimento implantado em dezembro de 2002, em Itamarandiba-MG. O material genético utilizado foi um clone de híbrido de Eucalyptus grandis x Eucalyptus camaldulensis. O delineamento experimental foi estabelecido em blocos ao acaso (três blocos), sendo testados cinco arranjos espaciais de 3,0 x 0,5 m; 3,0 x 1,0 m; 3,0 x 1,5 m; 3,0 x 2,0 m e 3,0 x 3,0 $\mathrm{m}$, denominados respectivamente de T1, T2, T3, T4 e T5. Cada parcela experimental foi constituída de seis linhas de plantio, cada uma com 28 covas, perfazendo um total de 168 indivíduos por tratamento e repetição.

Aos oito anos de idade foram abatidas 180 árvores, distribuídas em classes diamétricas, conforme a Tabela 1. Em cada árvore abatida foram medidas além do dap e da altura total ( $h t)$, os diâmetros com e sem casca nas seguintes posições: 0,10; 0,30; 0,70; 1,00; 1,30 e 2,00 m, e a partir deste ponto em intervalos de 1,0 em 1,0 m, até a posição onde $d=2,0 \mathrm{~cm}$ aproximadamente. O método de cubagem utilizado foi o proposto por Smalian, conforme Husch et al. (2003). 
Tabela 1. Distribuição de frequência das árvores abatidas aos oito anos de idade para cubagem em povoamentos de eucaliptos com diferentes arranjos espaciais, em Itamarandiba-MG.

Table 1. Frequency distribution of trees felled for scaling in stands of eucalyptus with different spacing in Itamarandiba-MG, in 2002.

\begin{tabular}{lccccc}
\hline \multirow{2}{*}{ Tratamentos } & \multirow{2}{*}{ Arranjo Espacial $(\mathbf{m})$} & \multicolumn{2}{c}{ Centro de Classe - dap (cm) } & \multirow{2}{*}{ Total } \\
\cline { 3 - 5 } & & $\mathbf{7 , 0}$ & $\mathbf{1 2 , 0}$ & $\mathbf{1 7 , 0}$ & 36 \\
T1 & $3,0 \times 0,5$ & 21 & 15 & - & 1 \\
T2 & $3,0 \times 1,0$ & 14 & 21 & 7 & 36 \\
T3 & $3,0 \times 1,5$ & 8 & 21 & 9 & 36 \\
T4 & $3,0 \times 2,0$ & 4 & 23 & 21 & 36 \\
T5 & $3,0 \times 3,0$ & - & 15 & 39 & 180 \\
\hline Total & & 47 & 94 & & \\
\hline
\end{tabular}

Com esses dados, ajustou-se os seguintes modelos de afilamento:

a) Modelo de Schöepfer (1966):

$$
\frac{d_{i}}{\text { dap }}=\beta_{0}+\beta_{1}\left(\frac{h_{i}}{\mathrm{ht}}\right)+\beta_{2}\left(\frac{h_{i}}{\mathrm{ht}}\right)^{2}+\beta_{3}\left(\frac{h_{i}}{\mathrm{ht}}\right)^{3}+\beta_{4}\left(\frac{h_{i}}{\mathrm{ht}}\right)^{4}+\beta_{5}\left(\frac{h_{i}}{\mathrm{ht}}\right)^{5}+\varepsilon
$$

b) Modelo de Garay (1979):

$$
\frac{d_{i}}{\text { dap }}=\beta_{0}\left(1+\beta_{1} \operatorname{Ln}\left(1-\beta_{2} h_{i}^{\beta_{3}} h t^{-\beta_{3}}\right)\right)+\varepsilon
$$

em que:

$d=$ diâmetro com casca na altura $h(\mathrm{~cm})$;

dap = diâmetro com casca $(\mathrm{cm})$, a 1,30 m de altura;

$h=$ altura comercial $(\mathrm{m})$;

$h t=$ altura total $(\mathrm{m})$;

$\beta_{\mathrm{k}}=$ parâmetros $(\mathrm{k}=0,1, \ldots, 5)$; e

$\varepsilon=$ erro estocástico, sendo $\varepsilon \sim \operatorname{NID}\left(0, \sigma^{2}\right)$.

Os ajustes referentes ao modelo linear foram feitos a partir do método dos mínimos quadrados ordinários e os referentes ao modelo não linear empregando o procedimento Gauss-Newton pelo método dos mínimos quadrados.

A qualidade dos ajustes foi avaliada individualmente por meio das estatísticas, coeficiente de correlação $\left({ }^{r} \hat{Y} Y\right)$, erro-padrão residual $\left(S_{\gamma x} \%\right)$, viés e análise gráfica dos resíduos. Considerou-se indispensável à análise gráfica dos resíduos, pois ela permite a avaliação de tendências nas estimativas além de detectar se os resíduos são independentes e se há homogeneidade na variância (CAMPOS; LEITE, 2013).

As equações de afilamento ajustadas a partir do modelo não linear foram comparadas empregando o teste de identidade de modelos, conforme Regazzi e Silva (2010). Da mesma maneira, as equações de afilamento ajustadas a partir do modelo linear foram comparadas empregando o teste de identidade de modelos, conforme Regazzi (1999).

A condição estabelecida para os testes de identidade de modelos são: rejeita-se $\mathrm{H}_{0}$ se e somente se $\mathrm{F}_{\text {Но }} \geq \mathrm{F}_{\alpha:(\mathrm{GL})}$, considerando uma probabilidade $\alpha=1 \%$, sendo GL o respectivo número de graus de liberdade.

\section{RESULTADOS E DISCUSSÃO}

As estimativas dos parâmetros e as estatísticas $r_{\hat{Y} Y}, S_{x y}$ e viés obtidos nos ajustes dos modelos de afilamento, para cada tratamento, são apresentadas na Tabela 2. É conveniente ressaltar que estas estatísticas não retratam o desempenho das funções de afilamento ao longo do fuste, tratam-se apenas, das médias das variações das estimativas totais. Logo, servem apenas como um indicativo da qualidade do ajuste. Assim, faz-se necessário analisar graficamente os resíduos e, também, validar os modelos em relação, principalmente, à variável d (CAMPOS; LEITE, 2013). 
Tabela 2. Estimativas dos parâmetros dos modelos de afilamento estimados em cada tratamento e as respectivas estatísticas empregadas para avaliar a qualidade dos ajustamentos.

Table 2. Estimates of the parameters of the taper models estimated in each treatment and the respective statistics used to evaluate the quality of the adjustments.

\begin{tabular}{|c|c|c|c|c|c|c|}
\hline \multirow{2}{*}{ Modelos } & \multirow{2}{*}{ Par./Est. ${ }^{(1)}$} & \multicolumn{5}{|c|}{ Tratamentos } \\
\hline & & T1 & T2 & T3 & T4 & T5 \\
\hline \multirow{9}{*}{$\begin{array}{l}\text { Schöepfer } \\
\text { (1966) }\end{array}$} & $\beta_{0}$ & $1,16856154^{* *}$ & $1,16220721^{* *}$ & $1,17568270^{* *}$ & $1,17664787^{* *}$ & $1,16763760^{* *}$ \\
\hline & $\beta_{1}$ & $-3,02245212^{\star *}$ & $-3,10010246^{\star *}$ & $-3,32502792^{\star *}$ & $-3,23011997^{\star *}$ & $-3,27135930^{* *}$ \\
\hline & $\beta_{2}$ & $13,17118389^{* *}$ & $13,75209369^{* *}$ & $14,77412849^{* *}$ & $14,02468618^{* *}$ & $14,22874988^{\star \star}$ \\
\hline & $\beta_{3}$ & $-32,89754500^{* *}$ & $-34,24138607^{\star *}$ & $-36,14636445^{\star *}$ & $-33,89100299^{\star *}$ & $-33,96062159^{* *}$ \\
\hline & $\beta_{4}$ & $36,21564865^{\star *}$ & $37,54061784^{\star *}$ & $39,21377461^{* *}$ & $36,48416905^{\star \star}$ & $35,94747372^{* *}$ \\
\hline & $\beta_{5}$ & $-14,61623940^{* *}$ & $-15,11999763^{* *}$ & $-15,71313837^{* *}$ & $-14,57199120^{* *}$ & $-14,11411271^{* *}$ \\
\hline & $r_{\hat{Y} Y}$ & 0,9907 & 0,9920 & 0,9925 & 0,9928 & 0,9924 \\
\hline & $\mathrm{S}_{\mathrm{xy}}(\%)$ & 4,8313 & 4,6336 & 4,4830 & 4,3728 & 4,4457 \\
\hline & Viés & $-9,83 \times 10^{-16}$ & $-2,64 \times 10^{-15}$ & $2,67 \times 10^{-15}$ & $-6,43 \times 10^{-15}$ & $-5,93 \times 10^{-15}$ \\
\hline \multirow{7}{*}{$\begin{array}{l}\text { Garay } \\
(1979)\end{array}$} & $\beta_{0}$ & 1,23934531 & 1,26818618 & 1,33183522 & 1,34581800 & 1,33236942 \\
\hline & $\beta_{1}$ & 0,35907258 & 0,29893995 & 0,26238211 & 0,24944384 & 0,25335241 \\
\hline & $\beta_{2}$ & 0,92016386 & 0,95765930 & 0,97379746 & 0,97870360 & 0,97882586 \\
\hline & $\beta_{3}$ & 0,31961824 & 0,24646873 & 0,17970019 & 0,16503318 & 0,16965867 \\
\hline & $r_{\hat{Y} Y}$ & 0,9916 & 0,9930 & 0,9946 & 0,9941 & 0,9945 \\
\hline & $\mathrm{S}_{x y}(\%)$ & 4,8482 & 4,6843 & 4,4746 & 4,3187 & 4,4492 \\
\hline & Viés & $-4,63 \times 10^{-9}$ & $-1,21 \times 10^{-9}$ & $1,36 \times 10^{-8}$ & $1,21 \times 10^{-8}$ & $3,09 \times 10^{-9}$ \\
\hline
\end{tabular}

Os gráficos de dispersão dos resíduos percentuais mostram a distribuição e o comportamento dos erros ao longo dos fustes. Analisando estes gráficos é possível perceber que os resíduos estão distribuídos uniformemente, o que indica boa precisão entre os valores estimados e observados, havendo concentração de pontos entre $\pm 10,0 \%$, em todos os tratamentos (Figura 1). Em todos os tratamentos houve maior dispersão dos resíduos localizados na região mais próxima à base dos fustes, de acordo com Souza et al. (2008a), isso ocorre pois na porção basal da árvore ocorre uma variação brusca no diâmetro.

O modelo de Garay foi o que melhor se ajustou aos dados, obtendo uma dispersão dos resíduos mais uniforme e agrupada, o modelo de Schöepfer, apresentou isoladamente alguns valores mais dispersos na região do topo dos fustes (Figura 1).

O modelo de Garay apresenta tendências comprovadas e fidedignas à realidade biológica para predição da forma dos fustes. Segundo Leite et al. (2006), este modelo é flexível o suficiente para descrever as variações de forma em árvores de diferentes espécies e tamanhos e consistente em relação às variáveis de diâmetro comercial, altura comercial e volume. O modelo de Schöepfer é um polinômio de grau elevado, capaz de descrever curvas com as mais variadas formas. No entanto, muitas vezes suas estimativas não apresentam uma tendência lógica quando avaliada do ponto de vista biológico. Horle et al. (2010), avaliando os ajustes de um modelo não linear (logístico modificado) e do modelo de Schöepfer, em árvores de Pinus oocarpa, concluíram, que o modelo não linear foi o mais indicado por apresentar uma melhor estabilidade e retratar as leis biológicas de crescimento.

A aplicação dos testes de identidade de modelos teve como propósito verificar se a forma (ou afilamento) dos fustes das árvores é coincidente para dois ou mais tratamentos. Os testes não linear e linear foram realizados para o conjunto de $n=4449$, pares de dados, e rejeitou-se $\mathrm{H}_{0}$ para $\alpha$ $=1 \%$, concluindo que pelo menos uma equação difere das demais, logo, um modelo comum $(\omega)$ não pôde ser utilizado para o conjunto total de dados, que envolve plantios em diferentes arranjos espaciais. Partiu-se então, para os testes aos pares de tratamentos para definição de quais os perfis diferem entre si.

Nas combinações avaliadas entre os pares de tratamentos nos testes não linear e linear, foi aceita a hipótese de nulidade $\mathrm{H}_{0}$ somente entre os tratamentos T1 $(3,0 \mathrm{x} 0,5)$ e T2 $(3,0 \mathrm{x} 1,0)$, conforme Tabela 3, indicando a igualdade entre as equações, portanto, a forma ou o afilamento dos fustes nestes tratamentos não diferiram entre si estatisticamente. 

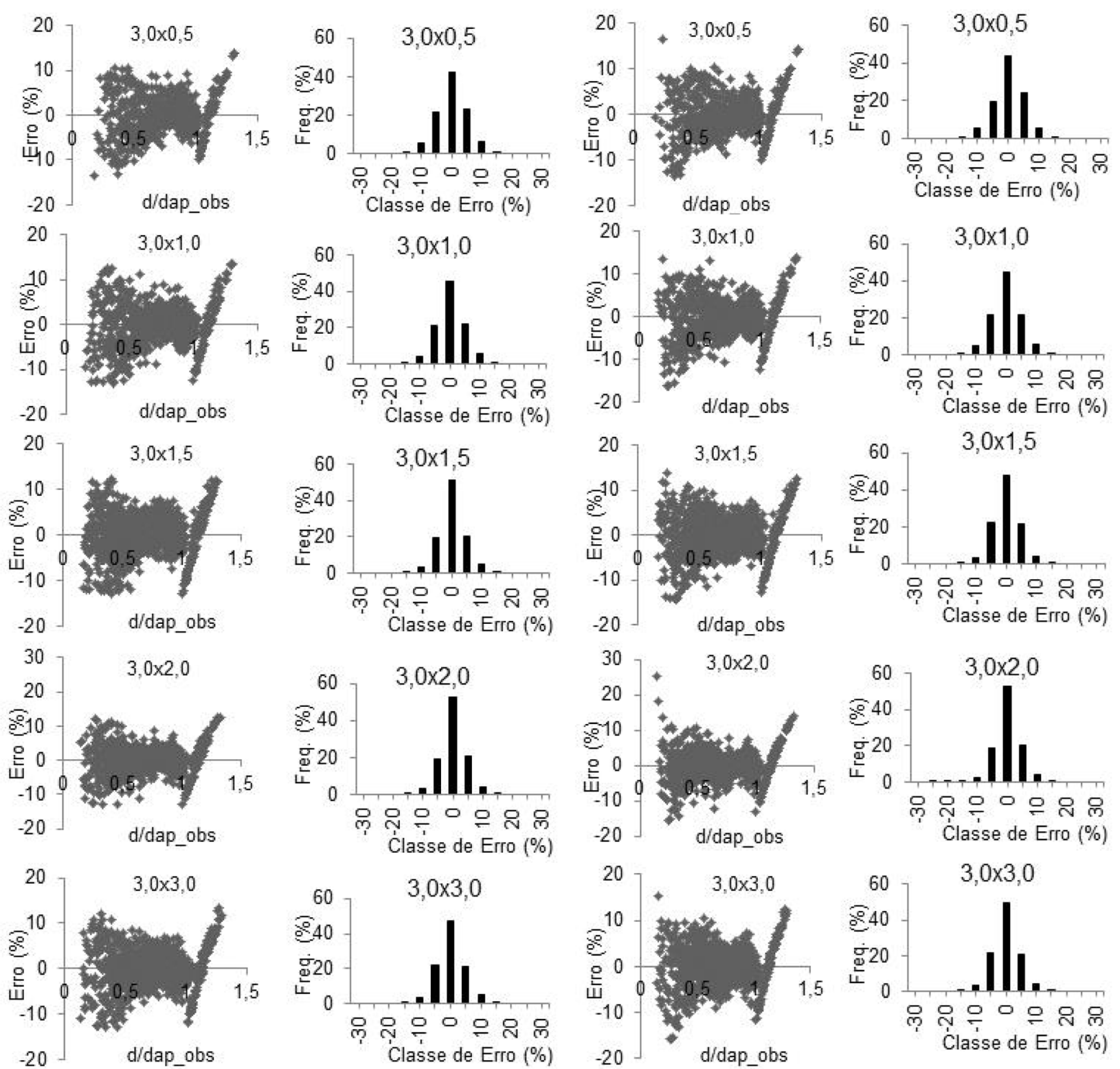

Figura 1. Erro percentual em função dos valores de d/dap observado e, frequência percentual dos erros de $d / d a p$ estimados, referentes aos ajustes dos modelos de Garay e de Schöepfer, em árvores de eucalipto em Itamarandiba-MG.

Figure 1. Percentage error in function of the values of $d / d a p$ observed and, percentage error frequency of $d / d a p$ estimated, referring to the adjustment of the models of Garay and Schöepfer, in eucalyptus trees in Itamarandiba-MG.

Tabela 3. Resultados ( $p$-valor) dos testes das hipóteses $H_{0}$ pela estatística $F$, referentes aos testes de identidade de modelo não linear e linear aplicados nas equações dos modelos de Garay e de Schöepfer, respectivamente.

Table 3. Results ( $p$-value) of tests of hypotheses $H_{0}$ for the $F$ statistic, referring to the identity test non-linear and linear models applied to equations of the Garay model and Schöepfer model, respectively.

\begin{tabular}{|c|c|c|c|c|c|c|c|c|}
\hline $\begin{array}{l}\text { Teste } \\
\text { Estatística } \\
\text { Modelo } \\
\end{array}$ & \multicolumn{4}{|c|}{$\begin{array}{c}\text { Não linear } \\
\text { p-valor }\left(F_{\mathrm{Ho}_{0}} \geq F_{\alpha}\right), \alpha=1,0 \% \\
\text { Garay }\end{array}$} & \multicolumn{4}{|c|}{$\begin{array}{c}\text { Linear } \\
\text { p-valor }\left(F_{H_{0}} \geq F_{\alpha}\right), \alpha=1,0 \% \\
\text { Schöepfer }\end{array}$} \\
\hline Tratamentos & $\mathrm{T} 2-3,0 \times 1,0$ & T3- 3,0 × 1,5 & $\mathrm{T} 4-3,0 \times 2,0$ & T5- 3,0 $\times 3,0$ & $\mathrm{~T} 2-3,0 \times 1,0$ & T3- 3,0 x 1,5 & T4- 3,0 × 2,0 & T5- 3,0 × 3,0 \\
\hline$T 1-3,0 \times 0,5$ & $0,0131^{* *}$ & $5,7783 \times 10^{-5}$ & $5,6708 \times 10^{-6}$ & $2,3245 \times 10^{-17}$ & 0,0078 & $9,8098 \times 10^{-5}$ & $2,4489 \times 10^{-35}$ & $2,2379 \times 10^{-24}$ \\
\hline T2- $3,0 \times 1,0$ & - & $5,8889 \times 10^{-38}$ & $1,3937 \times 10^{-10}$ & $4,3143 \times 10^{-12}$ & - & 0,0067 & $9,3952 \times 10^{-15}$ & 0,0003 \\
\hline T3- $3,0 \times 1,5$ & - & - & $6,7438 \times 10^{-42}$ & $2,9672 \times 10^{-51}$ & - & - & $1,1492 \times 10^{-107}$ & $2,2167 \times 10^{-76}$ \\
\hline T4- $3,0 \times 2,0$ & - & - & - & $1,1281 \times 10^{-22}$ & - & - & - & $2,4035 \times 10^{-21}$ \\
\hline
\end{tabular}

(I)** = significativo a $1 \%$ de significância.

(I) ${ }^{* *}=$ significant at $1 \%$ significance 
A Figura 2, ilustra o afilamento da árvore média estimado pelas equações dos modelos de Garay e de Schöepfer conforme o resultado dos respectivos testes de identidade de modelos. Os perfis descritos pelo modelo de Schöepfer apresentaram maior sinuosidade quando comparados aos descritos pelo modelo de Garay, provavelmente, pelo fato de se tratar de um polinômio de grau elevado, portanto muito mais flexível que o modelo de Garay.
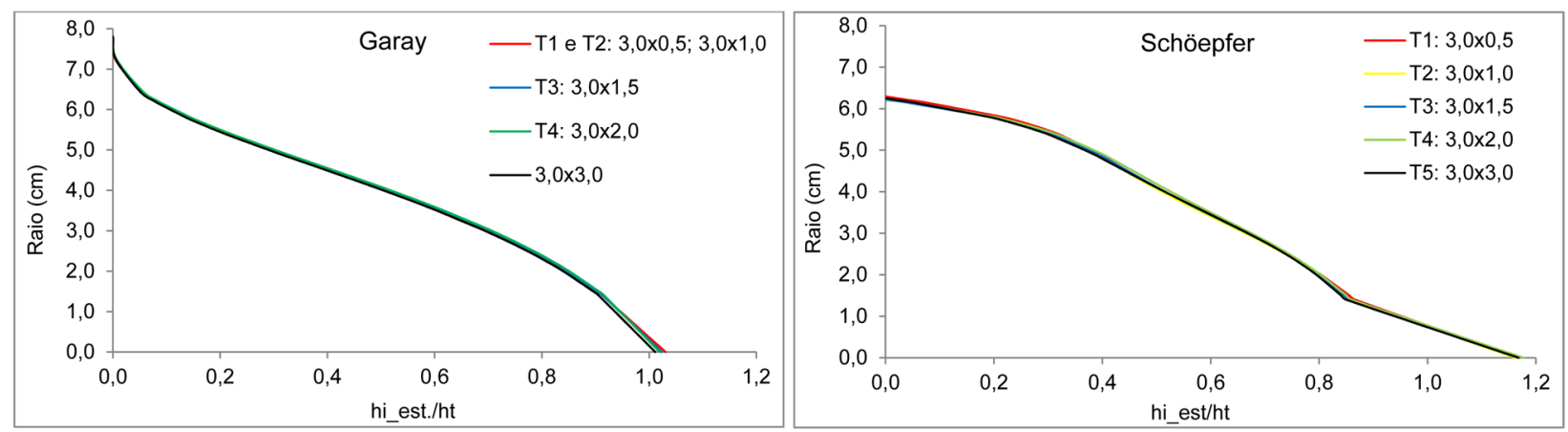

Figura 2. Estimativas dos perfis da árvore média (dap $=12,5 \mathrm{~cm} ; \mathrm{Ht}=25,0 \mathrm{~m}$ ), referentes aos ajustes dos modelos de Garay e de Schöepfer em diferentes arranjos espaciais, em Itamarandiba-MG.

Figure 2. Estimates of profiles of the average tree ( $\mathrm{dap}=12,5 \mathrm{~cm} ; \mathrm{Ht}=25,0 \mathrm{~m}$ ), referring to the adjustment of the models of Garay and Schöepfer in different spacing in Itamarandiba-MG.

O modelo de Schöepfer apresentou dificuldade em captar as diferenças nas formas dos fustes, estimando com menor precisão as regiões localizadas no topo dos fustes. É exatamente no topo que os fustes tendem a apresentar as maiores diferenças quando submetidos a arranjos espaciais muito reduzidos. Segundo Schneider (2008), em árvores médias ou dominadas sujeitas à competição intensa, há uma tendência de ocorrer um maior depósito de incremento nas posições superiores do fuste, de tal maneira que estas árvores adquirem uma forma mais cilíndrica. De acordo com Schonau e Coetzee (1989) devido ao aumento da densidade no povoamento ocorre uma redução do crescimento em diâmetro na parte inferior do fuste. Portanto, a utilização do modelo de Schöepfer para avaliar a forma não é adequada para as árvores deste estudo, entretanto, tal limitação não deve ser levada em consideração para as demais funções atribuídas ao referido modelo sem serem avaliadas. Segundo Campos e Leite (2013), a maioria dos modelos de afilamento não descrevem todo o fuste com a mesma precisão.

O modelo de Garay conseguiu estimar com precisão as variações ocorridas nas formas dos fustes. Segundo Leite et al. (2006), este modelo tem sido recomendado por vários estudos devido às suas propriedades estatísticas, resultando em estimativas quase sempre livres de tendências. Na ocasião, os autores relataram que Garay (1979) já havia demonstrado a eficiência desse modelo. Por ser derivado da função Chapman-Richards, este modelo é flexível o suficiente para descrever as variações de forma em árvores de diferentes espécies e tamanhos e consistente em relação às variáveis diâmetro $(d)$, altura ( $h$ ) e volume $(v)$. Souza (2009), concluiu em seu estudo, que, o desempenho do referido modelo foi melhor para descrever a forma de fustes com um, dois e três pontos de mudança de forma, quando comparado com um modelo segmentado. O referido autor, ainda salientou sobre a boa capacidade do modelo de Garay, em relação às árvores que apresentam três pontos de mudança na forma, pois, se tratando de fustes com as maiores variações quanto à forma, esperava-se que o modelo segmentado, justamente por considerar tais variações, fosse aquele com a maior acurácia. Leite et al. (2011), também observaram a superioridade do modelo de Garay quando comparado a outros dois modelos estatísticos, ao realizar estimativas do afilamento do cerne e do fuste em árvores de Tectona grandis.

Existe uma estreita relação entre o diâmetro da copa e o diâmetro da árvore (SCHONAU; COETZEE, 1989), sendo influenciada pela densidade do povoamento (GARBER; MAGUIRE, 2003). A disponibilidade e a competição por luz é afetada pelo arranjo espacial de plantio, assim espaçamentos que promovem intensa competição por luz produzem árvores com fustes mais finos, com menos e mais finas ramificações, e copas estreitas e pouco profundas (HENSKENS et al. 2001), e em consequência ao decréscimo das copas, os fustes apresentam formas mais cilíndricas (FRIEDL, 1989). Segundo Schonau e Coetzee (1989), este comportamento é devido ao Eucalyptus ser um gênero em que o inter-bloqueio dos ramos e a sobreposição das copas são raras, exceto em povoamentos mui- 
to jovens e densos, e que, o seu desenvolvimento também está sobre forte controle endógeno, dessa forma, a maioria dos Eucalyptus de crescimento rápido tem boa dominância apical, resultando em uma boa forma para o fuste.

Conforme Campos e Leite (2013) é esperado que, árvores conduzidas em menores arranjos espaciais iniciais, apresentem fustes mais cilíndricos, em comparação com árvores sob arranjos mais amplos. Esta característica também foi observada em Pinkard e Neilsen (2003), que avaliaram a influência de seis arranjos espaciais nas características da copa e do povoamento de Eucalyptus nitens para uma aplicação de desbaste na região norte da Tasmânia e em Nogueira et al. (2008), que avaliaram a influência do arranjo espacial sobre a forma do fuste de árvores de Pinus taeda. Rance et al. (2012), avaliando plantios de Eucalyptus grandis, submetidos a diferentes arranjos espaciais e conduzidos em distintos sítios, na região sudoeste da Austrália, observaram que, as referidas árvores já em idades juvenis apresentam fustes mais cilíndricos nos menores arranjos se comparados às árvores dos arranjos mais amplos. Os autores observaram que, as árvores menos ramificadas apresentavam fustes mais cilíndricos que os fustes das árvores mais ramificadas.

\section{CONCLUSÕES}

Dentre os modelos ajustados, o de Garay é indicado para descrever o afilamento de eucalipto nos arranjos espaciais avaliados (3,0 x 0,5 m; 3,0 x 1,0 m; 3,0 x 1,5 m; 3,0 x 2,0 m e 3,0 x 3,0 m).

A mesma equação pode representar a forma dos fustes em plantios com arranjos espaciais de 3,0 x 0,5 e $3,0 \times 1,0 \mathrm{~m}$.

\section{REFERÊNCIAS BIBLIOGRÁFICAS}

ANDRADE, C. M.; FINGER, C. A. F.; THOMAS, C.; SCHNEIDER, P. R. Variação do incremento anual ao longo do fuste de Pinus taeda L. em diferentes idades e densidades populacionais. Ciência Florestal, Santa Maria, v. 17, n. 3, p. 239-246, 2007.

CAMPOS, J. C. C.; LEITE, H. G. Mensuração florestal: Perguntas e respostas. 4.ed. Viçosa: UFV, 2013. 605 p.

FRIEDL, R. A. Dinâmica de forma dos fustes em povoamentos plantados de Araucaria angustifolia (Bert.) O. Ktze. 1989. 166 p. Dissertação (Mestrado em Ciências) - Universidade Federal do Paraná, Curitiba, 1989.

GARAY, L. Tropical forest utilization system. VIII. A taper model for entire stem profile including buttressing. Seattle: Inst. Forest Prod. Univ. Wash., 1979. 64 p. (Contrib. 36)

GARBER, S. M.; MAGUIRE, D. A. Modeling stem taper of three central Oregon species using nonlinear mixed effects models and autoregressive error structures. Forest Ecology and Management, Amsterdam, v. 179, n. 1-3, p. 507-522, 2003.

HENSKENS, F. L.; BATTAGLIA, M.; CHERRY, M. L.; BEADLE, C. L. Physiological basis of spacing effects on tree growth and form in Eucalyptus globulus, Trees, New York, v. 15, n. 6, p. 365-377, 2001.

HORLE, D. C.; MENDONÇA; A. R., CARVALHO, S. P. C.; CALEGARIO, N. Modelagem não linear do perfil longitudinal de fustes de Pinus oocarpa. Revista Cerne, Lavras, v. 16, n. 2, p. 177-184, 2010.

HUSCH, B.; BEERS, T. W.; KERSHAW JR., J. A. Forest mensuration. 4.ed. Malabar: Krieger Publishing Company, 2003. $443 \mathrm{p}$.

KOZAK, A.; MUNRO, D. D.; SMITH, J. H. G. Taper Function and their Aplications in Forest Inventory. Forest Chronicle, Quebec, v. 45, n. 4, p. 278-283, 1969.

LEITE, H. G.; GAMA, J. V.; CRUZ, J. P.; SOUZA, A. L. Função de afilamento para Virola surinamensis (ROLL.) WARB. Revista Árvore, Viçosa, v. 30, n. 1, p. 99-106, 2006.

Sci. For., Piracicaba, v. 44, n. 109, p. 33-40, mar. 2016

DOI: dx.doi.org/10.18671/scifor.v44n109.03 
Souza et al. - Forma de fuste de árvores de eucalipto em plantios com diferentes densidades iniciais

LEITE, H. G.; OLIVEIRA-NETO, R. R.; MONTE, M. A.; FARDIN, L.; ALCANTARA, A. M.; BINOTI, M. L. M. S.; CASTRO, R. V. O. Modelo de afilamento de cerne de Tectona grandis L.f. Scientia Forestalis, Piracicaba, v. 39, n. 89, p. 53-59, 2011.

MIGUEL, E. P.; MACHADO, S. A.; FIGUEIREDO FILHO, A.; ARCE, J. E. Modelos polinomiais para representar o perfil e o volume do fuste de Eucalyptus urophylla na região norte do estado de Goiás. Revista Floresta, Curitiba, v. 41, n. 2, p. 355-368, 2011.

NOGUEIRA, G. S.; LEITE, H. G.; REIS, G. G.; MOREIRA, A. M. Influência do espaçamento inicial sobre a forma do fuste de árvores de Pinus Taeda L. Revista Árvore, Viçosa, v. 32, n. 5, p. 855-860, 2008.

PINKARD, E. A.; NEILSEN, W. A. Crown and stand characteristics of Eucalyptus nitens in response to initial spacing: implications for thinning. Forest Ecology and Management, Amsterdam, v. 172, n. 2-3, p. 215-227, 2003.

PIRES, L. M.; CALEGARIO, N. Ajuste de modelos estocásticos lineares e não lineares para a descrição do perfil longitudinal de árvores. Revista Árvore, Viçosa, v. 31, n. 5, p. 845-852, 2007.

RANCE, S. J.; MENDHAM, D. S.; CAMERON, D. M.; GROVE, T. S. An evaluation of the conical approximation as a generic model for estimating stem volume, biomass and nutrient content in young Eucalyptus plantations. New Forests, Amsterdam, v. 43, n. 1, p. 109-128, 2012.

REGAZZI, A. J. Teste para verificar a identidade de modelos de regressão e a igualdade de parâmetros no caso de dados de delineamento experimentais. Revista Ceres, Viçosa, v. 46, n. 266, p. 383-409, 1999.

REGAZZI, A. J.; SILVA, C. H. O. Testes para verificar a igualdade de parâmetros e a identidade de modelos de regressão não linear em dados de experimento com delineamento em blocos casualizados. Revista Ceres, Viçosa, v. 57, n. 3, p. 315-320, 2010.

SCHNEIDER, P. R.; SCHNEIDER, P. S. P. Introdução ao manejo florestal. Santa Maria: FACOS-UFSM, 2008, 566 p.

SCHÖEPFER, W. Automatisierung des Massen, Sorten und Wertberechnung stenender Waldbestande Schriftenreihe Bad. [S.l.]: Wurtt-Forstl., 1966. n. p.

SCHONAU, A. P. G.; COETZEE, J. Initial Spacing, Stand Density and Thinning in Eucalypt Plantations. Forest Ecology and Management, Amsterdam, v. 29, n. 4, p. 245-266, 1989.

SOUZA, C. A. M. Modelos de afilamento para Pinus taeda L. ajustados segundo a forma do tronco e métodos de estratificação. 2009. 120 p. Tese (Doutorado em Engenharia Florestal) - Universidade Federal de Santa Maria, Santa Maria, 2009.

SOUZA, C. A. M.; SILVA, G. F.; XAVIER, A. C.; CHICHORRO, J. F.; SOARES, C. P. B; SOUZA, A. L. Avaliação de modelos de afilamento não segmentados na estimação da altura e volume comercial de fustes de Eucalyptus sp. Ciência Florestal, Santa Maria, v. 18, n. 3, p. 387-399, 2008 a.

SOUZA, C. A. M.; SILVA, G. F.; XAVIER, A. C.; CHICHORRO, J. F.; SOARES, C. P. B; SOUZA, A. L. Avaliação de modelos de afilamento segmentados na estimação da altura e volume comercial de fustes de Eucalyptus sp. Revista Árvore, Viçosa, v. 32, n. 3, p. 453-463, 2008b.

Recebido em 24/02/2015

Aceito para publicação em 30/07/2015 